\title{
Simulación clínica en enfermería comunitaria
}

\author{
Mariona Farrés Tarafa, Dolors Miguel Ruiz, Adrià Almazor, Esther Insa, Bárbara Hurtado, Carlos Nebot, \\ M. José Morera, M. Carmen Moreno Arroyo, David Bande, Juan Roldán Merino
}

Campus Docent Sant Joan de Déu (M. Farrés Tarafa, D. Miguel Ruiz,

A. Almazor, E. Insa, B. Hurtado, C. Nebot, M.J. Morera, J. Roldán Merino). Grupo de Investigación en Enfermería, Educación y Sociedad GIEES (M. Farrés Tarafa, D. Migue Ruiz, A. Almazor, E. Insa, B. Hurtado, C. Nebot, M.J. Morera, J. Roldán Merino). Escuela Universitaria de Enfermería del Campus de Bellvitge; Universidad de Barcelona (M.C. Moreno Arroyo). Parc Sanitari de Salut Mar (D. Bande)

Correspondencia: Mariona Farrés Tarafa. Sant Rosa, 39-57. E-08950 Esplugues de Llobregat (Barcelona).

E-mail: mfarres@santjoandedeu.edu.es (C) 2015 FEM
Introducción. La finalidad de esta investigación fue evaluar los resultados de aprendizaje del alumno en relación con las competencias en enfermería comunitaria y valorar la satisfacción de éstos respecto a la simulación clínica en este contexto.

Material y métodos. Estudio descriptivo observacional realizado en el primer semestre del curso 2014/2015 en el Campus Docent Sant Joan de Déu. Se hizo un análisis cualitativo de la conducción de 30 debriefings donde se evaluaron los resultados de aprendizaje en relación con los siguientes ítems (aspectos emocionales, toma de decisiones, valoración integral del paciente, valoración del paciente en atención domiciliaria, comunicación, educación sanitaria y habilidades técnicas). La satisfacción de los estudiantes se evaluó mediante un cuestionario compuesto por ocho ítems valorados mediante una escala ordinal (contenidos, coordinación, tiempo, metodología, utilidad, material, conocimientos y expectativas) y se realizó un análisis descriptivo de cada uno de ellos.

Resultados. El análisis de los debriefings mostró que los alumnos tienen dificultades para realizar la valoración del paciente con los instrumentos que se utilizan en la práctica clínica; sin embargo, presentan buenas habilidades comunicativas con el usuario y la familia. Respecto al grado de satisfacción, participaron en la cumplimentación del cuestionario 47 estudiantes de tercer curso de grado y la puntuación media total de los alumnos fue de $9,08 \pm 0,85$. La utilidad del taller fue valorada con una media superior a 9.

Conclusiones. La simulación clínica es una metodología docente valorada satisfactoriamente por parte de los alumnos que permite trabajar objetivos relacionados con habilidades técnicas y con habilidades no técnicas.

Palabras clave. Enfermería comunitaria. Habilidades no técnicas. Simulación clínica. Simulación con actores.

\section{Clinical simulation in nursing community}

Introduction. The purpose of this research was to evaluate the learning outcomes of students in relation to competencies in community nursing and rating the satisfaction regarding the clinical simulation in this context.

Material and methods. Descriptive observational study conducted in the first semester of the academic year 2014 / 2015 on the Campus Docent Sant Joan de Déu. A qualitative analysis of driving 30 debriefings where assessed learning outcomes in relation to the following items (emotions, decision-making, comprehensive patient assessment, assessing the patient in home care, communication, health education and technical skills) was made. Student satisfaction was assessed using a questionnaire consisting of 8 items valued using an ordinal scale (content, coordination, time, methodology, tool, material, knowledge, expectations) and we performed a descriptive of each analysis.

Results. The analysis of the debriefings showed that students have difficulties to conduct the assessment of the patient with the instruments that are used in clinical practice, however have good communicative skills with the user and the family. Regarding the degree of satisfaction, participated in filling in the questionnaire, 47 students in third year of degree, the total average score of students was $9.08 \pm 0.85$. The usefulness of the workshop was appraised with a superior half to 9 .

Conclusions. Clinical simulation is a valued teaching methodology successfully by students, which allows work related technical skills and abilities not technical objectives.

Key words. Clinical simulation. Community nursing. Not technical skills. Simulation with actors. 


\section{Introducción}

Las universidades europeas, en la última década, están experimentado importantes cambios durante el proceso de reforma de la educación superior, que pretende poner en común los distintos sistemas educativos entre los países del continente europeo, en el marco del Espacio Europeo de Educación Superior (EEES).

Esta transformación supone, entre otros cambios, la introducción de nuevas metodologías docentes cercanas a la realidad profesional y la reorganización de la educación en función del aprendizaje centrado en el alumno con la finalidad de desarrollar competencias como resultado del aprendizaje en la formación universitaria. Dentro del campo de la enfermería, se hace necesario introducir nuevas metodologías docentes dirigidas no sólo a evaluar conocimientos, sino también a evaluar habilidades y transmitir actitudes [1].

Debido a los múltiples cambios sufridos en los últimos años y entendiendo la necesidad de una educación basada en competencias, donde aparte de tener una definición teórica de las competencias para cada titulación es necesario evaluar las competencias adquiridas por los estudiantes, se introduce la simulación como una nueva metodología docente [2].

Los orígenes teóricos de la simulación se apoyan en el aprendizaje basado en la experiencia [3]. Existe una variedad de teorías del aprendizaje de educación que respalda el uso de la simulación en la formación de enfermería [4-6]. A finales de los años ochenta y principios de los noventa, se empieza a potenciar la idea de 'aprender a partir de la experiencia'. Según Donald Schön [7], el ciclo del aprendizaje tiene en cuenta la entrada de un conjunto de conocimientos que el profesional dispone fruto de la experiencia que ha ido acumulando con el tiempo para llegar a la zona de experto. Para llegar a ella, Schön [7] cita que es necesario el papel de un profesor facilitador o reflexivo, capaz de describir la acción y, en consecuencia, reflexionar sobre ella estableciendo un diálogo crítico entre profesor y estudiante. David Rodgers [4] habla de tres cualidades o actitudes que tienen que existir entre el profesor facilitador y el estudiante para que se dé la oportunidad de aprendizaje: la autenticidad, la valoración de la aceptación, la confianza del estudiante y comunicación de la comprensión empática.

No existe una definición consensuada sobre simulación clínica, y revisando literatura se encuentran múltiples definiciones. David Gaba lo define como 'Una técnica docente y no una tecnología, que se debe utilizar de una manera correcta, sin exagerar ni minimizar la realidad. Esta técnica pretende sustituir o ampliar las experiencias reales con experiencias guiadas que evocan o reproducen aspectos sustanciales del mundo real de una manera totalmente interactiva' [8]. La simulación clínica, para el Center for Medical Simulation (Cambridge, Massachusetts), 'es una situación o escenario creado para permitir que las personas experimenten la representación de un acontecimiento real, que puede ser cotidiano o, por el contrario, poco frecuente, pero no por esto irreal, con la finalidad de practicar, aprender, evaluar y adquirir conocimientos de sistemas o actuaciones humanas' [9]. Además, existen diferentes tipos de simuladores en función de los objetivos que se quieran alcanzar (a través de juegos de rol, con personas reales: actores, con maniquíes, a través de casos, de técnicas diagnósticas o terapéuticas, etc.); sin embargo, el objetivo general de toda simulación es involucrar a los estudiantes en el aprendizaje activo, el pensamiento crítico y la resolución de problemas [10].

Para desarrollar un taller de simulación, es necesario disponer de diferentes espacios: uno destinado al escenario donde se produce la situación y otro separado del anterior por espejos unidireccionales, dedicado a la sala de grabación. Finalmente, también es necesario un espacio destinado a la reflexión de la situación. En esta sala es donde se observa el desarrollo de la simulación a través de pantallas que muestran en tiempo real la monitorización del paciente y el desempeño de la situación por parte de los estudiantes; posteriormente, se realiza el debriefing.

El debriefing es probablemente lo más importante de esta técnica docente. Los propios estudiantes y el instructor/facilitador realizan un análisis guiado sobre el proceso de aprendizaje. Esto permite a los estudiantes ver, explicar, analizar y sintetizar la información y su estado emocional para argumentar o mejorar la actuación en similares situaciones futuras [11]. El momento óptimo para realizar el debriefing es inmediatamente después de completar el escenario, ya que tanto los alumnos como los instructores/facilitadores tienen el escenario y la experiencia vivida presente [12].

Las ventajas de utilizar la simulación como nueva metodología docente para la formación de enfermería son múltiples [13]. El empleo de la simulación acelera el proceso de aprendizaje del alumno y contribuye a elevar su calidad gracias a que se puede repetir el escenario tantas veces como necesite el estudiante hasta adquirir una habilidad. Además, permite practicar procedimientos clínicos complejos que pueden contribuir a que las habilidades 
Tabla I. Objetivos de aprendizaje.

\begin{tabular}{ll}
\hline Aspectos emocionales & $\begin{array}{l}\text { Tomar conciencia de la necesidad de gestionar las emociones } \\
\text { en situaciones de la práctica clínica }\end{array}$ \\
\hline Toma de decisiones & $\begin{array}{l}\text { Aprender a tomar la decisión adecuada en cada situación } \\
\text { después de valorar al usuario y el entorno }\end{array}$ \\
\hline $\begin{array}{l}\text { Valoración integral } \\
\text { del paciente }\end{array}$ & $\begin{array}{l}\text { Valorar al usuario y a su familia de forma integral y protocolizada } \\
\text { mediante instrumentos de valoración específicos }\end{array}$ \\
\hline $\begin{array}{l}\text { Valoración del paciente } \\
\text { en atención domiciliaria }\end{array}$ & $\begin{array}{l}\text { Valorar las actividades básicas de la vida diaria del usuario en } \\
\text { su entorno habitual y determinar las necesidades de éste y de su } \\
\text { cuidador/a principal mediante instrumentos de valoración específicos }\end{array}$ \\
\hline Comunicación & $\begin{array}{l}\text { Aprender los diferentes estilos comunicativos y adaptarlos } \\
\text { a las personas para cada situación }\end{array}$ \\
\hline Educación sanitaria & $\begin{array}{l}\text { Realizar educación sanitaria cuando sea necesario } \\
\text { tanto con el paciente como con el cuidador principal }\end{array}$ \\
\hline Habilidades técnicas & $\begin{array}{l}\text { Integrar las habilidades técnicas en una situación determinada } \\
\text { y en un entorno real simulado }\end{array}$ \\
\hline
\end{tabular}

Tabla II. Grado de satisfacción del estudiante respecto a la simulación.

\begin{tabular}{ll}
\hline Contenidos & $\begin{array}{l}\text { ¿Crees que en el taller de simulación se desarrollan todos los } \\
\text { contenidos de máximo interés para la valoración del paciente? }\end{array}$ \\
\hline Coordinación & $\begin{array}{l}\text { ¿Crees que la coordinación de la sesión, la logística del taller } \\
\text { y la dinámica han sido adecuadas? }\end{array}$ \\
\hline Tiempo & ¿Crees que el tiempo de la sesión ha sido el adecuado \\
\hline para obtener los objetivos preestablecidos?
\end{tabular}

aprendidas se puedan transferir a la realidad [14]. Asimismo, se adapta a las necesidades de aprendizaje del estudiante y proporciona un medio de entrenamiento de actitudes o habilidades no técnicas, como el trabajo en equipo, la comunicación, la toma de decisiones, el liderazgo o el pensamiento crítico entre otros [15]. Otro aspecto fundamental es que aumenta la seguridad de los pacientes al disminuir los errores humanos [16].

El objetivo de este estudio fue evaluar los resultados de aprendizaje del estudiante en relación con las competencias en enfermería comunitaria y valorar la satisfacción de éstos respecto a la simulación clínica en este contexto.

\section{Material y métodos}

Se realizó un estudio descriptivo observacional con dos tipos de análisis: uno cualitativo y otro cuantitativo.

El estudio se llevó a cabo en el centro de simulación del Hospital Sant Joan de Déu, donde se adaptó una sala a un entorno clínico idéntico a una consulta de enfermería de atención primaria y otra sala se adaptó a un domicilio, según el escenario que se pretendía simular. Por otro lado, también se utilizó la sala de debriefing, donde se reflexionó sobre dichos escenarios. Los casos que se trabajaron en el aula fueron simulados por dos actores del Institut del Teatre de Barcelona. Los aspectos que se abordaron en la simulación fueron previamente consensuados con los actores.

El estudio se realizó entre el mes de setiembre de 2014 y el mes de febrero de 2015. Se incluyó en el estudio a todos los estudiantes matriculados en la asignatura de Enfermería Comunitaria que aceptaron voluntariamente participar en el estudio mediante el consentimiento informado. Se excluyó a aquellos estudiantes que no se encontraron presentes el día que se realizó la simulación.

El análisis cualitativo se llevó a cabo en tres fases. En la primera fase se creó un grupo de discusión formado por tres profesores, dos expertos en enfermería comunitaria y uno experto en simulación. Se efectuó una primera reunión donde se establecieron siete objetivos de aprendizaje relacionados con las competencias en enfermería comunitaria (Tabla I) y se prepararon cinco casos a partir de estos objetivos.

En la segunda fase cada caso se repitió en seis grupos distintos de alumnos, por lo que se obtuvieron 30 debriefings con su correspondiente grabación. En esta fase participaron 60 estudiantes. Posteriormente, cada uno de los expertos visualizó las grabaciones individualmente.

En la tercera y última fase de esta etapa, se reunió el grupo de discusión para analizar las grabaciones y evaluar los resultados de aprendizaje del alumno.

Por otro lado, se realizó un análisis cuantitativo para valorar la satisfacción del alumno respecto a la simulación en enfermería comunitaria. Se utilizó un formulario ad hoc compuesto por ocho ítems (Tabla II) valorados mediante una escala ordinal 
(donde 1 corresponde a poco satisfecho y 10 a muy satisfecho). El cuestionario se administró una vez finalizada la sesión de simulación. Se hizo un análisis descriptivo de cada una de las variables incluidas en el estudio. Para el análisis de los datos se empleó el paquete estadístico SPSS v. 17.0. Se trabajó con un nivel inferior o igual a 0,05 .

\section{Resultados}

\section{Aprendizaje del estudiante según las competencias en enfermería comunitaria trabajadas con la simulación}

Respecto a los aspectos emocionales, se identificó que los estudiantes tomaron conciencia de las emociones aparecidas durante la atención a los usuarios y de la dificultad que tenían para gestionarlas. El debriefing ayudó a dichos estudiantes a identificar nuevas estrategias que favorecían la adecuada gestión emocional. Por otro lado, los estudiantes tuvieron dificultad para realizar una valoración objetiva del usuario o cuidador mediante la utilización de instrumentos de valoración específicos. El debriefing les ayudó a tomar conciencia de la dificultad que tenían para tomar decisiones adecuadas sin una valoración previa del paciente.

Respecto a la comunicación, los estudiantes demostraron tener una comunicación efectiva tanto con el usuario como con los acompañantes o cuidadores principales, empleando diferentes estilos comunicativos según la persona, el entorno o la situación.

A pesar de que los estudiantes identificaron correctamente los casos en los que se debía realizar una educación sanitaria, encontraron dificultades para explicar el funcionamiento de material médico específico o algunas técnicas por una falta de conocimientos teóricos previos.

En las habilidades técnicas, a pesar de que los estudiantes siguieron de forma adecuada el protocolo de actuación frente a la realización de algunas técnicas enfermeras específicas, demostraron dificultad para mantener la comunicación con el usuario durante dicho procedimiento. El debriefing ayudó a los estudiantes a encontrar soluciones, como informar al usuario antes de la realización del procedimiento para mantener una mayor concentración durante su ejecución.

Los resultados de aprendizaje del estudiante en relación con cada una de las competencias identificadas en la enfermería comunitaria se representan en la tabla III.
Tabla III. Resultados de aprendizaje.

\begin{tabular}{ll}
\hline Aspectos emocionales & $\begin{array}{l}\text { Los alumnos toman conciencia de sus emociones y aprenden } \\
\text { estrategias para gestionarlas }\end{array}$ \\
\hline $\begin{array}{l}\text { Toma de decisiones } \\
\text { Valoración integral } \\
\text { del paciente }\end{array}$ & $\begin{array}{l}\text { Los alumnos reconocen la importancia de realizar una buena } \\
\text { valoración del paciente para tomar la decisión adecuada }\end{array}$ \\
\hline $\begin{array}{l}\text { Valoración del paciente } \\
\text { en atención domiciliaria }\end{array}$ & $\begin{array}{l}\text { Los alumnos muestran dificultad para valorar al usuario o cuidador/a } \\
\text { principal mediante instrumentos de valoración específicos para atención } \\
\text { domiciliaria }\end{array}$ \\
\hline Comunicación & $\begin{array}{l}\text { Los alumnos integran la necesidad de adoptar un estilo comunicativo } \\
\text { adaptado a cada persona, a su situación y entorno }\end{array}$ \\
\hline $\begin{array}{l}\text { Educación sanitaria } \\
\text { Habilidades técnicas }\end{array}$ & $\begin{array}{l}\text { Los alumnos identifican cuándo se tiene que realizar educación sanitaria } \\
\text { protocolo correcto, pero muestran dificultad para integrar las habilidades } \\
\text { técnicas en una situación determinada }\end{array}$ \\
\hline
\end{tabular}

\section{Grado de satisfacción de los estudiantes \\ respecto a la simulación clínica en la \\ asignatura de Enfermería Comunitaria}

Participaron en la cumplimentación del cuestionario 47 estudiantes de tercer curso de Grado en Enfermería matriculados en la asignatura de Enfermería Comunitaria, 15 de mañana y 32 de tarde. La puntuación media total de los estudiantes de mañana fue de $8,82 \pm 0,96$ y la de los estudiantes de tarde fue de 9,34 $\pm 0,74$. Las variables mejor puntuadas por el grupo de mañana fueron la utilidad del taller de simulación y la metodología docente utilizada, que obtuvieron una puntuación de 9,0 \pm 0,7 y 9,1 \pm 0,78 , respectivamente. Sin embargo, el grupo de tarde valoró con mayor puntuación la utilidad del taller de simulación y las expectativas de éste, con $9,64 \pm 0,81$ y $9,51 \pm 0,74$, respectivamente.

\section{Discusión}

Múltiples estudios [2,4-6,17] describen la importancia de utilizar simulación para trabajar las competencias enfermeras. En todos ellos se concluye que aprender a través de la simulación ayuda al estudiante a integrar no sólo habilidades técnicas, sino también otras habilidades no técnicas tan importantes como la toma de decisiones, el trabajo en equipo y la comunicación. Hay pocos estudios que describan resultados de la experiencia de simula- 
ción en enfermería comunitaria; sin embargo, un estudio de la Universidad de Carolina del Norte [18] describe la satisfacción de 76 estudiantes después de realizar un escenario sobre el paciente domiciliario. La media de satisfacción de los estudiantes respecto a las expectativas del taller fue valorada con un 4,67 en una escala del 1 al 5. Otro estudio de los mismos autores [19] describe la satisfacción de 50 alumnos después de realizar escenarios de simulación de enfermería comunitaria por pares, a través de una encuesta online con una puntuación del 1 al 5. Según el estudio, los alumnos perciben que mejoran en la valoración del paciente con una media de 4,52 \pm 0,76, 4,46 $\pm 0,76$ en comunicación, $4,53 \pm 0,68$ en pensamiento crítico, 4,46 $\pm 0,73$ en conocimientos técnicos y 4,49 $\pm 0,73$ en el rendimiento general. Por lo tanto, los resultados del presente estudio concuerdan con los resultados de otros estudios que evalúan la satisfacción de los estudiantes después de realizar simulación en enfermería comunitaria.

\section{Conclusión}

Los resultados de este estudio concluyen que el $d e-$ briefing que se realiza en la simulación clínica permite a los alumnos aprender mediante el pensamiento crítico y reflexionar sobre la práctica clínica. Además, la simulación clínica es una metodología docente valorada satisfactoriamente por parte de los alumnos que permite trabajar objetivos relacionados con habilidades técnicas y no técnicas.

\section{Conceptos básicos}

- Realizar simulación con un paciente simulado (actor) es efectivo para trabajar habilidades no técnicas con estudiantes de enfermería.

- El debriefing que se realiza en la simulación clínica permite a los alumnos aprender mediante el pensamiento crítico y reflexionar sobre la práctica clínica.

- La simulación clínica en enfermería comunitaria es una metodología docente valorada satisfactoriamente por parte de los alumnos.

\section{Bibliografía}

1. Agencia Nacional de Evaluación de la Calidad y Acreditación Libro blanco. Título de Grado de Enfermería. URL: www. aneca.es/media/150360/libroblanco_jun05_enfermeria.pdf. [05.05.2015].

2. Cant RP, Coper SJ. Simulation-based learning in nurse education: systematic review. J Adv Nurs 2010; 66: 3-15.

3. Cioffi J. Clinical simulations: development and validity. Nurse Educ Today 2001; 21: 477-86.

4. Rodgers DL. High-fidelity patient simulation: a descriptive white paper report. URL: http://sim-strategies.com/ downloads/simulation\%20white\%20paper2.pdf. [05.05.2015].

5. Rothgeb MK. Creating a nursing simulation laboratory: a literature review. J Nurs Educ 2008; 47: 489-94.

6. Parker BC, Myrick F. A critical examination of high-fidelity human patient simulation within the context of nursing pedagogy. Nurse Educ Today 2009; 29: 322-9.

7. Schön D. La formación de profesionales reflexivos. Hacia un nuevo diseño de la formación y el aprendizaje de profesionales. 3 ed. Madrid: Paidós-MEC; 1992.

8. Gaba DM. The future vision of simulation in healthcare. Simul Healthc 2007; 2: 126-35.

9. Del Moral I, Díaz de Terán C, Rabanal J, Quesada A. Nuevos procedimientos de entrenamiento en el manejo de crisis y emergencias médicas. En Quesada A, Rabanal JM, eds. Procedimientos técnicos en urgencias y emergencias. $2 \mathrm{ed}$. Madrid: Ergon; 2003. p. 479-87.

10. McCaughey $\mathrm{C}$, Traynor $\mathrm{K}$. The role of simulation in nurse education. Nurse Educ Today 2010; 30: 827-32.

11. Simon R, Rudolph J, Raemer D. Debriefing assessment for simulation in healthcare: rater version. Cambridge, MA: Center for Medical Simulation; 2009.

12. Tood M, Manz JA, Hawkins KS, Parsons ME, Hercinger M. The development of a quantitative evaluation tool for simulations in nursing education. Int J Nurs Educ Scholarsh 2008; 5: 1-17.

13. Yuan HB, Williams BA, Fang JB, Ye QH. A systematic review of selected evidence on improving knowledge and skills through high-fidelity simulation. Nurse Educ Today 2012; 32: 294-8.

14. Stayt LC. Clinical simulation: a sine qua non of nurse education or a white elephant? Nurse Educ Today 2012; 32: e23-7.

15. Moule P. Simulation in nurse education: past, present and future. Nurse Educ Today 2011; 31: 645-6.

16. Quesada A, Buron FJ, Castellanos A, Del Moral I, González C, Olalla JJ, et al. Training in the care of the critical and multipleinjured patient: role of clinical simulation. Med Intensiva 2007; 31: 187-93.

17. Sharpnack PA, Goliat L, Rogers K. Using standardized patients to teach leadership. Clinical Simulation in Nursing 2013; 9: e95-102.

18. Kim-Godwin YS, Rivers K, Ezzell D, Highmith C. Home visit simulation using a standardized patient. Clinical Simulation in Nursing 2013; 9: e55-61.

19. Kim-Godwin YS, Rivers K, Ezzell D, Highmith C, Ashley N, Winslow $\mathrm{H}$, et al. Students like peer evaluation during home visit simulation experiences. Clinical Simulation in Nursing 2013; 9: e535-42. 